\title{
Die Geschichte der Kinder- und Jugendpsychiatrie in Österreich
}

\section{Der Versuch der Zusammenschau einer langen Entwicklung}

\author{
Leonhard Thun-Hohenstein
}

Eingegangen: 29. April 2017 / Angenommen: 23. Juni 2017 / Online publiziert: 30. August 2017

(c) Der/die Autor(en) 2017. Dieser Artikel ist eine Open-Access-Publikation.

\begin{abstract}
Zusammenfassung Die Geschichte unseres Faches in Österreich im engeren Sinne ist erst sehr kurz - seit 1975 respektive 2007, allerdings wurden in den Zeiten davor im Namen der Kinder- und Jugendpsychiatrie unter dem Deckmantel höchsten wissenschaftlichen Interesses Kinder und Jugendliche ihren Eltern weggenommen, gequält und getötet, weil sie als „unwertes Leben" befunden worden waren. Auch die Geschichte nach 1945 zeigt, wie stark bestimmte Ideen und Vorstellungen in den Menschen weiterleben und wir daher täglich dazu angehalten sind, unsere Handlungen auf Menschlichkeit zu überprüfen und auf die Menschenrechte zu beziehen.

In den letzten zehn Jahren hat sich unser Fach in Österreich hervorragend entwickelt, die Versorgung ist wesentlich besser geworden und auch Lehre, Wissenschaft und Aus- und Weiterbildung sind dramatisch ausgebaut worden. Die Fachgesellschaft hat sich - obwohl ausschließlich ehrenamtlich geführt - professionalisiert und wird daher politisch und gesellschaftlich entsprechend wahrgenommen.
\end{abstract}

Schlüsselwörter Historische Entwicklung - Kinderund Jugendpsychiatrie

\footnotetext{
L. Thun-Hohenstein $(\bowtie)$

Univ.-Klinik für Kinder- und Jugendpsychiatrie,

Universitätsklinikum Salzburg, SALK-PMU, Ignaz

Harrerstraße 49, 5020 Salzburg, Österreich

L.Thun-Hohenstein@salk.at
}

The history of child and adolescent psychiatry in Austria

Essay about a long development

Abstract The history of child and adolescent psychiatry in Austria is very short - it was established first in 1975 , finally it achieved the status of a seperate specialisation in 2007. In earlier years the name of this specialty was used under the guise of scientific interest for malpractice and child abuse or child murder during the times of the 2nd World War.

But also, the development after 1945 showed how long abviant ideas could survive. During the last decade CAP showed a formidable development, quality and support of patient care improved significantly. Also the scientific development and education in CAP improved. The Austrian Society for CAP developed - although exclusively privately run - into a professional and scientific society, which ist respected by politicians and society.

Keywords Historic development - Child and Adolescent Psychiatry

\section{Einleitung}

Das Sonderfach Kinder- und Jugendpsychiatrie (infolge KJP genannt) wurde in Österreich erst 2007 durch die neue Ärzteausbildungsordnung ins Leben gerufen. Das hatte einen ausgeprägten Entwicklungsschub zur Folge, doch gerade dadurch wurden die vorhandenen Mängel des Faches noch deutlicher. Bei aller Freude über die Schaffung dieses Faches und der folgenden Ereignisse gilt es aber auch sich der Geschichte des Faches und seiner Vorläufer zu stellen. Castell [14] formulierte dies in seinem Buch so: ,jede Wissenschaft, 
die Bestand haben will, muss sich ihrer Geschichte bewusst sein" - oder es gilt, wie dies Frank Häßler \& Reis formulieren: „Die Erinnerung an die Geschichte als Mahnung für die Gegenwart“ [28] zu verstehen.

\section{Periode 1: Jahrhundertwende bis zum National- sozialismus}

\section{Geschichtlicher Hintergrund}

Die Jahrhundertwende in Wien - wie auch in den übrigen Großstädten Europas - war eine Zeit rasanter und oft gegenläufiger Entwicklungen. Das damals aufkommende „liberale Bekenntnis im weitesten Sinne forderte von seinen Anhängern die Überantwortung des Herzens, des Verstandes und des Willens an eine Welt, die durch rationale regulative Grundsätze geordnet war" [42]. Es war eine Zeit des Umbruchs, einerseits eines ausgeprägten Liberalismus, der besonders in Wissenschaft, Kunst und Kultur seinen Ausdruck fand [52] und andrerseits eine Zeit des Nationalismus und Rassismus, durchaus ein Erbe der Gegenreformation.

1859 hatte Darwin seine Evolutionslehre publiziert, die in der Folge von verschiedenen Forschern für andere Gebiete verwendet wurde: in seiner „Natürlichen Schöpfungsgeschichte“ (1868) interpretiert der Naturforscher Ernst Häckel (1843-1919) den Kampf des Einzelnen ums Dasein auf die Völker um und nützt dafür auch die Theorie der „natürlichen Auslese“. 1895 publiziert der englische Physiologe John B. Haycraft (1859-1922) „Darwinism and Race Progress“ und 1904 gründet der Arzt und Sozialist Alfred Plötz die „Gesellschaft für Rassenhygiene“. Die Entwicklung dieser „eugenischen“ Theorien wird in der Geschichte der KJP noch eine wichtige Rolle spielen. Eugenik wurde nach Sir Francis Galton (britischer Naturforscher und Schriftsteller; 1822-1911) definiert als „die Wissenschaft von allen Einflüssen, die die angeborenen Eigenschaften einer Rasse verbessern“ und unter „alle Einflüsse" versteht er Theorien aus den Bereichen des Sozialdarwinismus, Rassismus und der erbbiologischen Lehre [4].

In diese Zeit fällt auch die Entdeckung des Individuums und der Seele als Objekt der Wissenschaft [32] und damit auch des Kindes als eigenständiges Wesen. Die Kulturgeschichtlerin Ellen Key [30] ruft das „Jahrhundert des Kindes“ aus und verarbeitet in ihrer weithin beachteten Publikation darwinistische und im weitesten Sinne auch eugenische Theorien. Die Pädagogin Adele Schreiber [43] verfasst ihr „Buch vom Kinde“, indem sie versucht, den Aristotelischen Gedanken „Alle Erziehung darf nur Ergänzung der Natur sein" mit wissenschaftlichen Erkenntnissen und gemeinsam mit vielen Ko-Autor_innen laiengerecht $\mathrm{zu}$ formulieren, um so die Erziehung des Kindes im Allgemeinen zu verbessern. Auch die Medizin interessiert sich zunehmend für das Kind wie z. B. Hans Spitzy's Buch „Die körperliche Erziehung des Kindes“
[46] zeigt. Siegmund Freud (1856-1939) begründet mit seiner „Traumdeutung“ 1900 in Wien die Psychoanalyse und schafft so die Grundlage für die Entwicklung der Psychotherapie. Die Ärztin Maria Montessori (1870-1952) gründet ihre Casa Bambini (1907) und publiziert ihre erste Schrift (Il metodo della pedagogia scientifica 1909), in der sie ihre - Montessori-Pädagogik genannte - Methode und deren Anwendung beschreibt und damit einen wesentlichen Grundstein für eine neue Pädagogik legt.

\section{Die Anfänge der Kinderpsychiatrie}

Das erste KJP-Fachbuch wird 1867 von Henry Maudsley (1835-1918, Insanity of early life) in England und im deutschen Sprachraum 1887 von Hermann Emminghaus (1845-1904; „Die psychischen Störungen des Kindesalters“) veröffentlicht. Der Begriff „Kinderpsychiatrie“" wurde 1899 durch den französischen Psychiater Marcel Manheimer-Gommés (1870-1936) eingeführt [16, 53]. Leo Kanner, ein großer amerikanischer Kinderpsychiater, sieht in seiner „History of child psychiatry“ $[29,36]$ die Entstehung der KJP als Folge von drei wesentlichen Gegebenheiten im Umkreis der Jahrhundertwende 19./20. Jahrhundert: der Einführung der Psychometrie (z. B. die erste Version des Stanford-Binet Intelligenz Test 1905), der Fortschritte der Psychiatrie (z. B. werden die Leistungen Emil W. Kraeplins (1856-1926) als Beispiel angeführt) und die „Bewegung für Mental Health“, deren amerikanischer Zweig 1909 gegründet wurde. Als erste klinische Einrichtungen für Kinderpsychiatrie werden die Provinzial-Kinderanstalt für seelisch Abnorme in Bonn von Otto Löwenstein (1889-1965; [51]) bzw. die Kinderabteilung der Nervenklink in Tübingen angeführt [15]. An letztgenannter Abteilung wurde Werner Villinger [12] ausgebildet, der später im Rahmen des T4-Programmes eine wichtige Rolle spielen sollte.

\section{Heilpädagogik}

Eine ganz eigene Entwicklung wurde 1890 durch die Gründung des ersten Heimes für entwicklungsgeschädigte und -gestörte Kinder (Sophienhöhe in Jena) durch Johannes Trüper (1855-1921) eingeleitet, welches als erste heilpädagogische Einrichtung gilt [9]. Die Urväter des Namens „Heilpädagogik“ sind die deutschen Reformpädagogen Jan Daniel Georgens (1823-1886) und Heinrich M. Deinhardt (1821-1880), fußend auf dem „Lehrbuch für allgemeine Erziehungskunde“ von Vinzenz E. Milde (1777-1855). Sie definierten Heilpädagogik als die Theorie der Erziehung behinderter und/oder abnormaler Personen (zit n. [9]). In Österreich entsteht 1911 die Heilpädagogische Station an der Universitätskinderklinik Wien, gegründet von deren Leiter, Clemens von Pirquet (1874-1929), der die Leitung dieser Station dem Kinderarzt Erwin Lazar (1877-1932) übertrug. Der Begriff Heilpädagogik wurde in Österreich in der Folge eher 
für eine medizinische Spezialität verwendet und später auch von Hans Asperger (1906-1980) für seine Psychopathologie des Kindes [1] verwendet.

Die heilpädagogische Station als erste österreichische Vorläuferstation der heutige Kinder- und Jugendpsychiatrie wurde von 1911-32 von Lazar geleitet. Bis 1932 war sie eine klassische Beobachtungsstation mit 15 Betten. Ziel der Aufnahme war die Erstellung einer Prognose über die Persönlichkeit des jugendlichen „Missetäters oder Verwahrlosten“. Die ursprünglich soziale Kategorie der Verwahrlosung wurde durch die Übernahme durch die Psychiatrie medikalisiert, und es wurden diese Begriffe in der Folge zu einem Rechtsbegriff [38], welcher später als Grundlage für die T4Aktion dienten. Die Heilpädagogische Station war also anfangs ausschließlich beobachtend und diagnostisch - also begutachtend - tätig und keine Heilanstalt. Im Zentrum von Lazars Forschung standen Fragen nach den Auswirkungen von Anlage- und Milieufaktoren bei der Entstehung psychischer Erkrankungen. Er vertrat ein für damalige Begriffe modernes multifaktorielles Verständnis der Pathogenese und sorgte in seiner Funktion als Konsulent für Heilpädagogik im Staatsamt für Volksgesundheit u. a. auch dafür, dass in den verschiedenen Einrichtungen das ungelernte Personal durch PädagogInnen ersetzt wurde [9]. Nach dem Tod Lazars wurde 1932 zuerst Valerie Bruck und ab 1935 Hans Asperger als Arzt an die Heilpädagogische Station berufen [5]. Er schuf dort einen Lern- und Spielhort, der nun das bisherige ausschließlich diagnostische Angebot um (heil-) pädagogische Maßnahmen erweiterte [9], gleichzeitig übernahm er auch die von Lazar begonnenen Betreuung der Wiener Kinder- und Jugendfürsorge.

\section{Periode 2: Nationalsozialismus bis 1945}

1937 fanden drei wichtige internationale Kongresse, wegen der Weltausstellung, in Paris statt: der 1. Internationale Kongress für Kinderpsychiatrie, der 2. Internationale Kongress für psychische Hygiene und der 11. Internationale Kongress für Psychologie [13]. An diesen nahmen auch einige deutsche Psychiater teil, auch Ernst Rüdin (1874-1952). An allen drei Kongressen war dieser geladener Redner und vertrat dort die eugenische Rassenhygiene als eine Maxime der psychiatrischen Arbeit [13]. Er war Psychiater und genoss seine Ausbildung bei Eugen Bleuler (1857-1939) und Emil W. Kraeplin (1856-1926). Er war Mitbegründer und Redakteur des von Alfred Plötz herausgegebenen Archivs für Rassen- und Gesellschaftsbiologie sowie Gründungsmitglied der Gesellschaft für Rassenhygiene. Er war zur Zeit des Nationalsozialismus der Vorsitzende der Deutschen Gesellschaft für Neurologie und Psychiatrie, in deren Wissenschaftlichen Beirat der spätere 1. Präsident der Deutschen Gesellschaft für Heilpädagogik und Kinderpsychiatrie, Paul Schröder saß [41]. Rüdin war wesentlich an dem Zustandekommen des „Gesetzes zur Verhütung erbkranken Nachwuchses“ vom Juli 1933 beteiligt, welches wiederum die Grundlage für die T-4-Euthanasie-Aktion bildete. Gemeinsam mit Alfred Plötz, Fritz Lenz und Hans F.K. Günther war er Teil einer 1933 durch Reichsinnenminister Wilhelm Frick ins Leben gerufenen Arbeitsgemeinschaft für Rassenhygiene und Rassenpolitik [14].

1938 kam es zum „Anschluss“ Österreichs an HitlerDeutschland und damit zur Übernahme aller Staatsgeschäfte durch die Nationalsozialisten.

Am 01.09.1939 ermächtigte Adolf Hitler auf Basis des 1933 beschlossenen „Gesetzes zur Verhütung
Abb. 1 Versuch derRekonstruktion des Netzwerkes der T4-Aktion als Folge des Euthanasiebefehls vonAdolf Hitler auf Basis der angegebenen Literatur

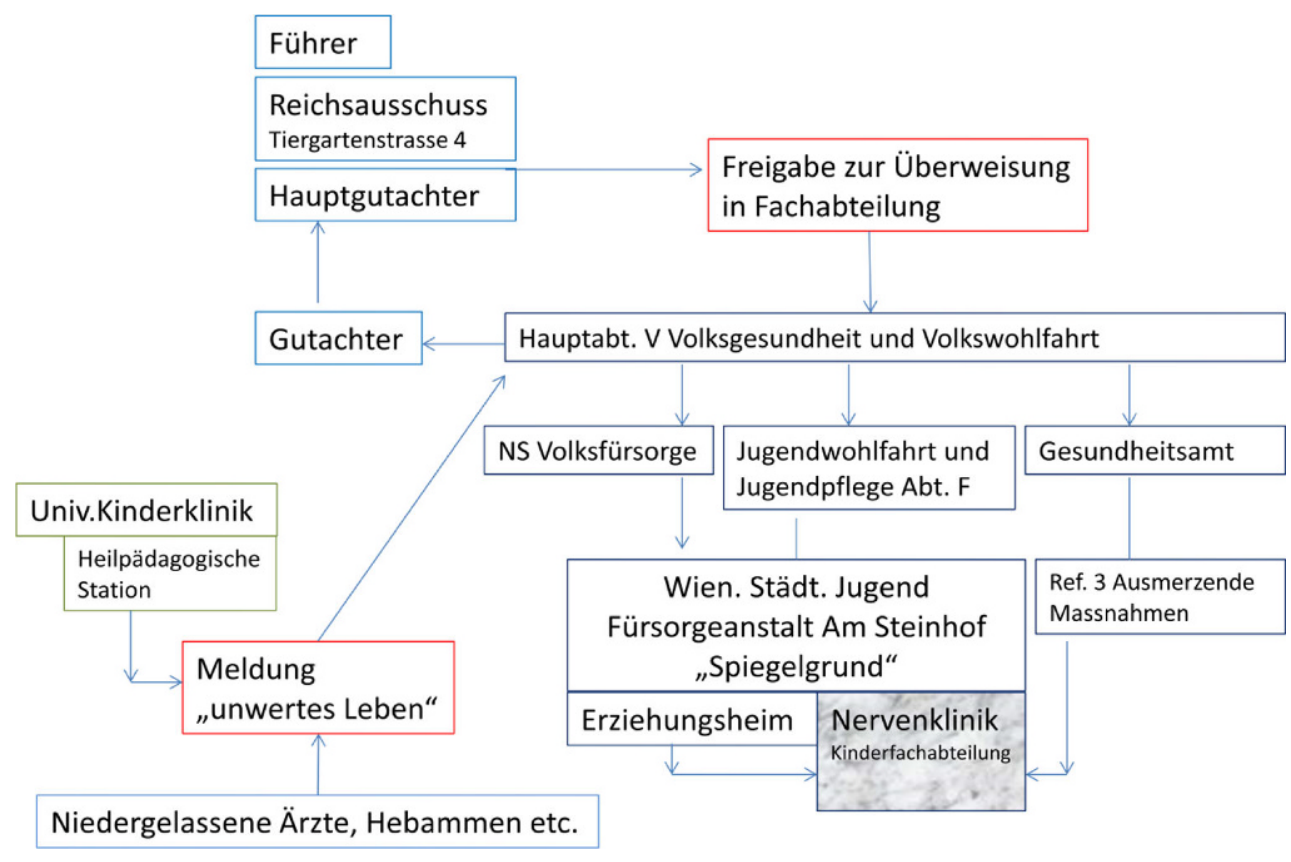


erbkranken Nachwuchses“ „unheilbar Kranken“ den Gnadentod zu „gewähren“ [21, 24]. Ebenfalls 1939 erging eine - als geheime Reichssache deklarierte Meldepflicht für alle „missgestalteten usw. Neugeborene“. Der Entartungsbegriff wurde immer weiter ausgedehnt und inkludierte auch psychische Devianzen und soziale Benachteiligung („Verwahrlosung“). Es entstand ein streng administrativ durchorganisiertes Netzwerk (s. Abb. 1) bestehend aus der Kanzlei des Führers, den Fürsorge-, Gesundheits- und Wohlfahrtsämtern, Heimen, Hebammen und niedergelassenen Ärzten und vielen mehr [18, 24, 40]. Zumeist angeschlossen an psychiatrische Kliniken wurden sogenannte „Kinderfachabteilungen“ - insgesamt über 30 - gegründet, mit dem Zweck, die dem „Reichsausschuss“ gemeldeten und von den Gutachtern als „lebensunwert“ befundenen Kinder zu töten. Zwei dieser „Kinderfachabteilungen“ befanden sich in Österreich: die „Klinik am Spiegelgrund“ und der „Feldhof“ in Graz [24]. Mehr als 40 Psychiater waren in diesem Netzwerk als Gutachter und drei weitere als Obergutachter tätig, sieben Personen davon aus Österreich [21]: zwei sind für die Geschichte der KJP wichtig: Hans Bertha, Primarius für Neurologie und 1944/45 amtierender Direktor Am Steinhof und der Kinderarzt Erwin Jekelius, der 1931-1936 an der Heilpädagogischen Station tätig und 1940-1941 ärztlicher Direktor der Wiener städtischen Fürsorgeanstalt „Am Spiegelgrund“ war [35]. Auch Heinrich Gross erhielt 1941 eine Ausbildung an der Wiener Kinderklinik [18]. 1940 eröffnete die Stadt Wien die Wiener städtische Jugendfürsorgeanstalt „Am Spiegelgrund“, unterteilt in eine Nervenklinik (Kinderfachabteilung) und ein Erziehungsheim [33]. 789 Kinder wurden im Pavillon 15 ermordet [18], die sterblichen Überreste sind am Zentralfriedhof 2002 in einem Ehrengrab der Stadt Wien beigesetzt worden.

Aufgrund des ausgeprägten kirchlichen Widerstandes [21] wurde die T4-Aktion 1941 beendet, aber Ärzte erhielten auch weiterhin den Auftrag unwertes Leben zu beenden. Diese - sogenannten dezentralen Anstaltsmorde („wilde Euthanasie“; [21]) dauerten noch zumindest bis zum Ende des Zweiten Weltkrieges an.

\section{Gründung der deutschen Fachgesellschaft}

1939 wurde in Wiesbaden eine Arbeitsgemeinschaft für Kinderpsychiatrie gegründet, die die Gründung der Deutschen Gesellschaft für Kinderpsychiatrie und Heilpädagogik vorbereiten sollte [41]. Diese Gründung fand während der 1. Tagung der Deutschen Gesellschaft für Kinderpsychiatrie und Heilpädagogik 1940 in Wien statt. Im Rahmen dieses Kongresses sprachen prominente Vertreter der deutschen Kinderpsychiatrie wie P. Schröder (1. Vorsitzender der neuen Fachgesellschaft) und W. Villinger und aus Wien der Wiener Klinikvorstand der Kinderklinik, F. Hamburger [13, 41], der sich als Pädiater wissenschaftlich auch mit psychischen Erkrankungen beschäftigte [25].

\section{Die Heilpädagogik in der nationalsozialistischen Ära}

Die Leitung hatte der Kinderarzt Hans Asperger, anfangs arbeitete auch noch Jakelius an dieser Station. Der politische Hintergrund zog tiefe Spuren in die Universitätskliniken, 80 \% der Ärzte wurden aufgrund ihrer jüdischen Abstammung entlassen und nur die politisch genehmen oder unauffälligen durften bleiben. Nach Zeitzeugenberichten müssen diese verbliebenen Kollegen - so sie nicht selbst Nazi waren - einem großen Druck ausgesetzt gewesen sein, Nationalsozialistischen Organisationen wie der NSDAP oder NSD-Ärztebund beizutreten. Asperger schien nie als aktiver Nationalsozialist auf, er setzte sich so gut er konnte für „seine“ Patienten ein [3, 37, 44], dennoch wurden auch einige Kinder von der Heilpädagogischen Station und der Universitäts-Kinderklinik an den Spiegelgrund transferiert [18]. Nach Czech $[17,18]$ kann kein Zweifel an der Bedeutung einer Transferierung an den Spiegelgrund bestanden haben, wie aus Arztbriefen und anderen Dokumenten zu entnehmen war. 1943 habilitierte Asperger über die „autistischen Psychopathien“ [2]. Asperger war in dieser Zeit auch als Gutachter für die Nationalsozialistische Volkswohlfahrt (NSV) tätig, gleichzeitig mit Erwin Jekelius, der als Leiter der „Fürsorge für Geisteskranke, Psychopathen und Süchtige“ aktiv war. Die NSV war wiederum Teil des großen Netzwerkes, das unter anderem die Umsetzung des Reichsauschussbeschlusses von 1939 durchzuführen hatte.

\section{Periode 3: 1945-1975}

Mit Ende des Zweiten Weltkriegs und den Nürnberger Prozessen wurde einigen Nazi-Ärzten der Prozess gemacht, aber einige konnten entkommen. So konnten viele von ihnen als anerkannte medizinische Autoritäten lange Zeit in der österreichischen Gesellschaft eine wesentliche Rolle spielen siehe z. B. Heinrich Gross.

Für die Entwicklung der Kinder- und Jugendpsychiatrie in dieser Zeit postuliert Berger [5], dass die Entwicklung der KJP in dieser Periode von drei großen Linien geprägt war:

1. Heilpädagogisch-repressive Perspektive

2. eine segregative Behindertenmedizin und -pädagogik

3. eine sozialpsychiatrisch-psychotherapeutische Perspektive

Ad 1)

Hans Asperger versuchte zwischen 1949 und 1955 mit allen Kräften ein Extraordinariat für Heilpädagogik zu schaffen, was aber sowohl der eigene Chef (Karl Kundratitz (1889-1975)) und das Professorenkollegium abgelehnt hatten als auch im Ministerium keinen Erfolg nach sich zog. Eine Rolle spielte auch der Streit zwischen Asperger und Hans Hoff (ab 1950 Vorstand der Universitätsklinik für Neurologie und Psychiatrie; 
1897-1969), der selbst den Aufbau einer Kinderstation plante. Asperger wurde dann 1957 als ordentlicher Professor für Kinderheilkunde nach Innsbruck berufen und kehrte 1962 als Leiter der Wiener Kinderklinik wieder zurück. Er widmete sich auch als Klinikchef noch der Weiterentwicklung der Heilpädagogik. Ausgehend von der Heilpädagogischen Station in Wien entstanden in mehreren Bundesländern ähnliche Stationen. Die Heilpädagogik war auch nach Ende des 2. Weltkrieges lange Zeit in einem stark biologistischen Denken verhaftet, Diagnosen wurden wie Etiketten verwendet und hatten zukunftsweisende Konsequenzen. Die aufgenommenen Patient_innen wurden in uniforme Bekleidung gekleidet, es galten sehr strenge Formen - was aber insgesamt gesellschaftlich $\mathrm{zu}$ dieser Zeit nicht außergewöhnlich schien. Nur sehr langsam setzte sich eine individualistische und entwicklungsorientierte Haltung durch. An manchen heilpädagogischen Einrichtungen hielten sich diese Ideen und Haltungen noch sehr lange. Mittlerweile liegen zahlreiche Studien vor [34, 39, 45, 49], die die Auswirkungen der biologistisch und rassisch-eugenisch basierten Denkweise auf die österreichische Nachkriegszeit mehr als deutlich belegen und somit auch die Heilpädagogische Station nach 1945 und ihre Nachfolgeeinrichtungen (siehe auch ein publiziertes Beispiel [31]) zu einem Gutteil als biologistisch-eugenischer Nachhall gesehen werden müssen.

\section{Ad 2)}

Vor wenigen Monaten ist der - nach Berichten im FALTER von der Stadt Wien und dem Wiener Krankenanstaltenverbund beauftragte - Bericht des Forschungsteams des Instituts für rechts- und Kriminalsoziologie - „Kinder und Jugendliche mit Behinderung in der Wiener Psychiatrie zwischen 1949-1985“ publiziert worden [34]. Darin wird der Umgang mit behinderten Kindern und Erwachsenen im NachkriegsWien bis in die späten 80er Jahre beschrieben. Hemma Mayerhofer (S. 14) schreibt dort wortwörtlich: „Pavillon 15 stellte ein umfassendes Gewaltsystem dar, das über den gesamten Zeitraum (1949-1983/84) für die überwiegende Anzahl der Kinder völlig inadäquate Versorgungs- und Betreuungsverhältnisse aufwies“. Wesentlicher Grund für die Einweisung der Mehrzahl der Kinder mit Behinderungen oder sozialen Auffälligkeiten waren fehlende Versorgungsstrukturen für diese Altersgruppe außerhalb der Psychiatrie. 1956 wurde „Abteilung für Entwicklungs-gestörte Kinder“ im Altersheim Lainz gegründet und ab 1975 in einem neuerrichteten Pavillon im Neurologischen Krankenhaus Rosenhügel verortet. Einerseits war diese Einrichtung modern und richtungsweisend für die Versorgung behinderter Kinder, gleichzeitig aber auch höchstproblematisch und von einem stark biologistisch geprägten Menschenbild geprägt [39]. Der Leiter, Andreas Rett (1924-1997) - Kinderarzt, Beschreiber des Rett-Syn- droms und früheres NSDAP-Mitglied - war ein charismatischer Mensch und bemüht, den Menschen medizinische Hilfe und Fürsorge zuteilwerden zu lassen, ging aber davon aus, dass Behinderung eine lebenslange Versorgungs- und Therapiebedürftigkeit nach sich ziehe. Eigenwillige Medikationen (Epiphysan zur Unterdrückung des Sexualtriebs), Zwangssterilisation von überwiegend weiblichen Patientinnen, Entscheidungen über Schwangerschaftsabbrüche waren Standard. Die zunehmende Emanzipationsbewegung und Bestrebungen nach Selbstbestimmung, die ab Anfang der 80er Jahre immer stärker wurden, lehnte Rett ab und hielt an seinem Modell der segregierten Behindertenbetreuung fest [34]. Die Ideen und Praktiken von Andreas Rett waren zu dieser Zeit äußerst öffentlichkeitswirksam und fanden viele Unterstützer und stille Nachahmer.

\section{Ad 3)}

Zum Glück wurde diese Haltung nicht an allen Heilpädagogischen Einrichtungen und nicht von allen dort tätigen Menschen gelebt. An anderen Heilpädagogischen Abteilungen begann sich im Gegensatz dazu eher die sozialpsychiatrisch-psychotherapeutische Richtung durchzusetzen [47, 48]. Hans Asperger selbst hat die Entwicklung hin zu einer psychotherapeutischen Heilpädagogik und damit zur Kinderpsychiatrie in den Anfängen erlebt, selbst nicht mehr aufgenommen, aber jungen Kolleg_innen eine Entwicklung in diese Richtung ermöglicht (siehe Film „Heilpädagogisches Sommerlager 1980“ Reisetbauer/ Thun-Hohenstein [44]). Tatzer [48] beschäftigt sich in dieser Zeit mit Systemischer und Familientherapie und spätestens ab der Übernahme der Leitung der Wiener Heilpädagogischen Station durch Werner Leixnering 1993 hat sich die psychotherapeutische Sichtweise als Grundprinzip durchgesetzt.

Ab 1949 wurde an der psychiatrisch-neurologischen Universitätsklinik mit einem „Kinderzimmer“ der Ausbau der klinisch-psychiatrischen Kinderpsychiatrie begonnen. Diese Entwicklung mündete - über einige Zwischenstufen - in der Schaffung einer eigenen Universitätsklinik für Kinder- und Jugendneuropsychiatrie im Jahre 1975, deren erster Ordinarius Walter Spiel (1920-2003) war. Diese Entwicklung konkurrenzierte die bisherige Monopolstellung der Heilpädagogik im Wiener Raum und damit verlor die Heilpädagogik zunehmend an Bedeutung. Die schon zwischen Asperger und Hoff begonnen Streitigkeiten zwischen diesen beiden Bereichen haben leider noch lange Jahre die dort arbeitenden Kolleginnen und die Fachgesellschaft nachhaltig beschäftigt. Diese Animositäten waren aus heutiger Sicht in hohem Maße der unklaren Haltung und Tätigkeit Aspergers während der Nazi-Zeit zuzuschreiben und andererseits der politischen Herkunft der beiden Konkurrenten geschuldet. Asperger entstammte konservativen Kreisen und Walter Spiel sozialdemokratischen Kreisen. 
Nach Ernst Berger [5] entwickelte Walter Spiel an seiner Klinik eine sozialpsychiatrisch-psychotherapeutisch orientierte Kinderpsychiatrie, er übernahm auch zunehmend die Themenführung im Bereich der Jugendwohlfahrt und Jugendgerichtsbarkeit. Doch trotz dieser Neuausrichtung der Kinder- und Jugendpsychiatrie ist die Situation des Pavillon 15 und der Rett-Klinik erst sehr spät und nur aufgrund einer engagierten Krankenschwester ans Licht gekommen [50]. Ausgehend von der Abteilung für Kinder- und Jugendpsychiatrie des AKH-Wien entwickelten sich in den Bundesländern ebenfalls kinder- und jugendpsychiatrische Stationen (z. B. Feldkirch ab 1976 oder Salzburg ab 1983).

\section{Periode 4: 1975-2007}

1975 wurde auch in der österreichischen Ärzteausbildungsordnung eine neue Ära begonnen, es wurde der Zusatzfacharzt für Kinder- und Jugendneuropsychiatrie etabliert, der es Fachärzten aus den Mutterfächern Pädiatrie, Neurologie und Psychiatrie nach Abschluss ihrer Grundfachausbildung ermöglichte, in 3 Jahren das Zusatzfach zu erwerben. Als Ausbildungsstätten wurden in einer Übergangsregelung alle bestehenden heilpädagogischen und kinderpsychiatrischen Einrichtungen akzeptiert, als Ausbildner alle einschlägig arbeitenden KollegInnen. Es wurde im selben Jahr auch die Fachgesellschaft für Kinder- und Jugendneuropsychiatrie gegründet, deren erster Präsident Walter Spiel wurde.

Diese Zeit war eine Zeit der Identitätsfindung unseres Faches, in der unausgesprochen die Nachwirkungen der Vergangenheit zu spüren waren. Die Konflikte zwischen den Klinikchefs Asperger und Spiel, die sich auf die MitarbeiterInnen der beiden Kliniken, aber auch auf die Fachgesellschaft ausweiteten, waren wichtige energetische Impulse zur Findung einer gemeinsamen Identität, die letztlich in den Bemühungen zu einem gemeinsamen eigenständigen Fach Kinder- und Jugendpsychiatrie mündeten. Ein wichtiger Streitpunkt war der Verlust des „Neuro“ im Facharzt gewesen, die für einige Kinder- und Jugendpsychiater selbstverständlich zum Fach gehörte, wie dies von Gerhard Göllnitz (1920-2003) in die Kinder- und Jugendpsychiatrie eingeführt worden war und in einigen Nachbarländern auch üblich war (DDR) und ist (Italien).

In dieser Zeit hat sich die Kinder- und Jugendpsychiatrie in Österreich zu einer modernen und humanen Fachrichtung entwickelt mit einer klaren, gesetzlichen Definition: „Das Sonderfach Kinder- und Jugendpsychiatrie und Psychotherapeutische Medizin umfasst die Prävention, Diagnostik, Behandlung einschließlich Psychotherapeutischer Medizin und Rehabilitation von im Kindes- und Jugendalter auftretenden psychischen Krankheiten, Störungen und Verhaltensauffälligkeiten einschließlich der psychiatrischen Behandlung von entwicklungsbedingten psychischen Erkrankungen sowie die fachspezifische Begutachtung" [10]. Schon 2006 hatten die Vertreter der österreichischen Kinder- und Jugendpsychiatrie [7] entsprechende Versorgungsstrukturen eingefordert, die allerdings weder von der Quantität noch von der geforderten Qualität bis heute erreicht wurden (siehe Beitrag [27] in diesem Heft).

\section{Periode 5: 2007-2017}

Die Psychotherapie hielt an allen Kinder- und Jugendpsychiatrischen Einrichtungen Einzug und wird heute als ein wesentlicher Grundpfeiler der fachlichen Qualität und Qualifizierung angesehen. Dies hatte zur Folge, dass die Ausbildung für Psychotherapeutische Medizin in die Facharztausbildung [11] integriert wurde und der Facharzt nun FA für Kinder- und Jugendpsychiatrie und Psychotherapeutische Medizin heißt [11]. Für die Abteilungen bedeutet dies eine massive Anpassungsleistung und Veränderung der Ausbildung an die neuen Gegebenheiten. Die Fachgesellschaft hat sich in diesem Zusammenhang das Ziel gesetzt, den Kolleg_innen in Ausbildung die Theorie der Psychotherapeutischen Medizin in einem durch die Fachgesellschaft organisierten und von den Ländern finanzierten Curriculum anzubieten.

Mit der Schaffung dieses eigenständigen Faches Kinder- und Jugendpsychiatrie waren die Länder und der Bund erstmals gezwungen, sich um die flächendeckende Versorgung zu kümmern. In Tab. 1 sind die Entstehungsdaten aller österreichischen Abteilungen für Kinder- und Jugendpsychiatrie zusammengetragen. 6 der 11 Abteilungen wurden als Folge der Facharzteinführung etabliert.

Nach einer zweijährigen Probephase [26] wurde auch die Niederlassung des Sonderfaches Kinderund Jugendpsychiatrie genehmigt und mit Kassenverträgen ausgestattet (siehe auch [27] in diesem Heft).

Die Zusammenarbeit mit den öffentlichen Stellen wurde wesentlich verbessert, so gibt es regelmäßige Expertentreffen im Rahmen der Gesundheit Österreich $\mathrm{GmbH}$, mit dem Hauptverband der Sozialversicherungen, der Ärztekammer und anderen wesentlichen Stakeholdern im Gesundheitswesen. Die Einführung des Faches zog auch wesentliche Veränderungen in der Fachgesellschaft nach sich. Es musste eine standardisierte und qualitätsgesicherte Facharztprüfung erarbeitet und jedes Jahr gewährleistet werden, wofür die Prüfungskommission gegründet wurde. Weiters wurden eine Ausbildungskommission und eine Qualitätssicherungskommission gegründet, alle Kommissionsvorsitzenden haben Sitz und Stimme im Vorstand. Die Fachgesellschaft beteiligt sich ebenfalls an den Aktivitäten der Politischen Kindermedizin (www.polkm.at).

Eine aktuelle Arbeitsgruppe beschäftigt sich mit dem Unterbringungsgesetz und dem Umgang mit Zwangsmaßnahmen, was insbesondere vor dem historischen Hintergrund unseres Faches ein besonders 
\|\|\|\|\|\|||||

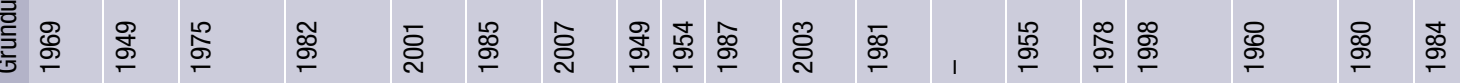

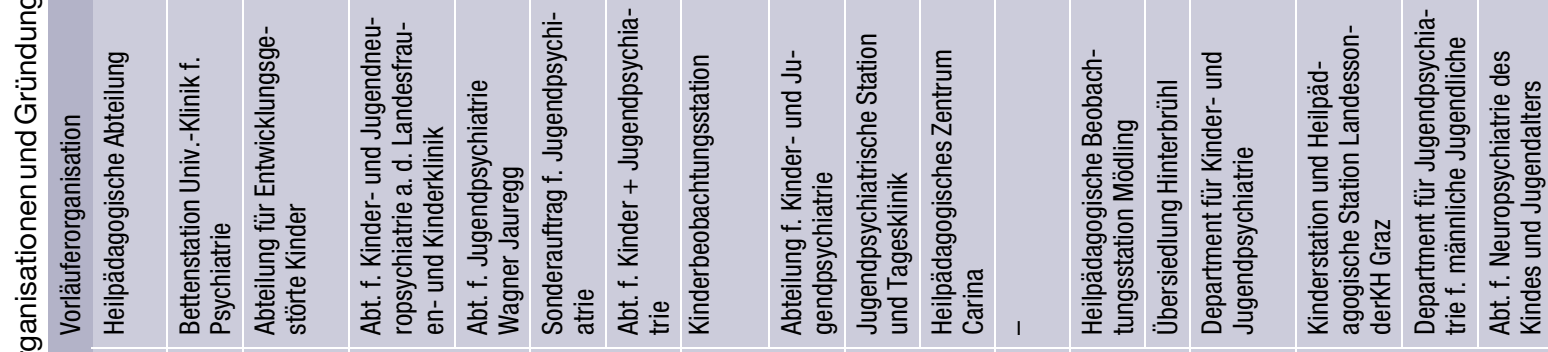

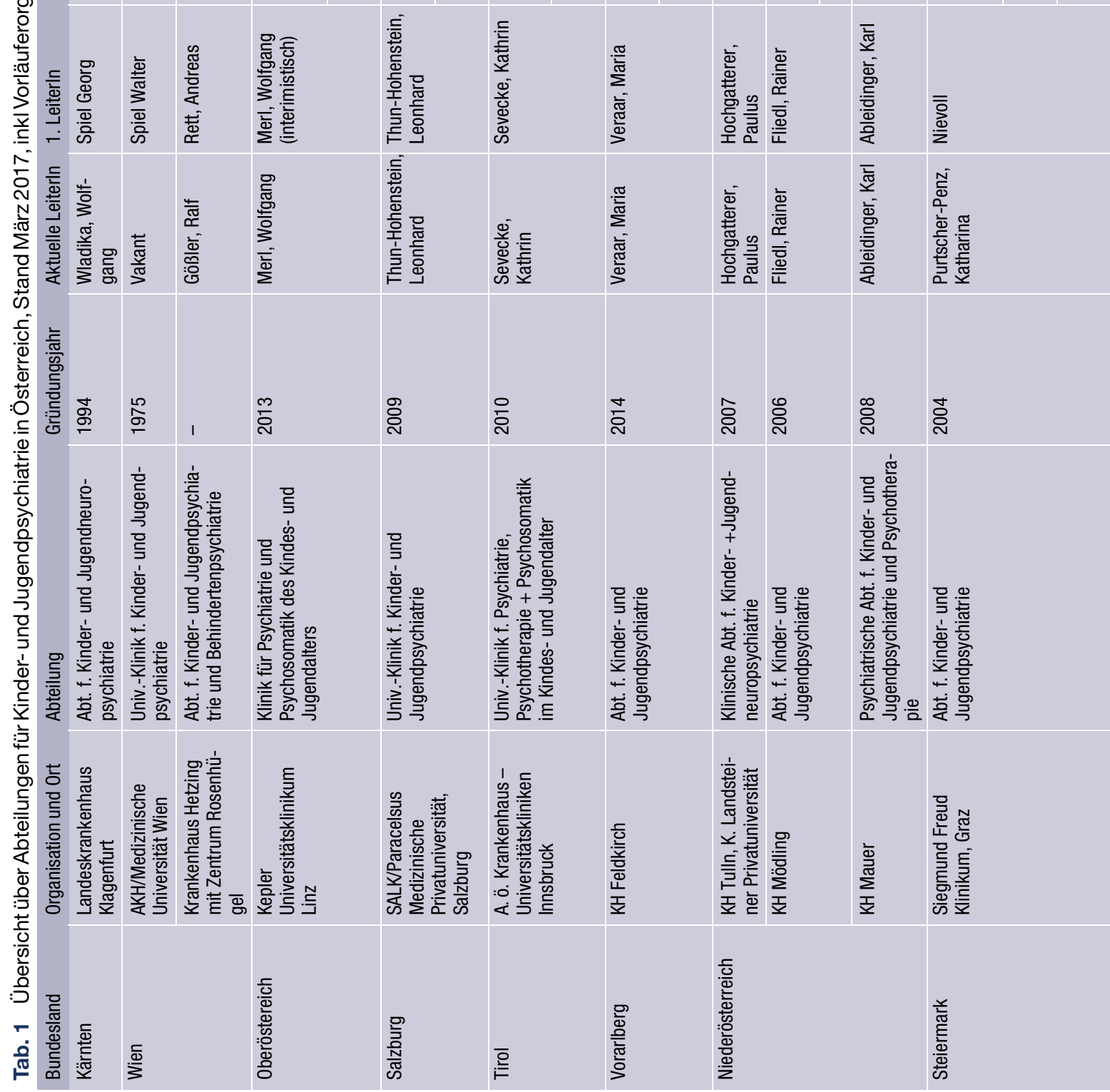


heikles Thema darstellt. Basis der Neuformulierung von eigenen, alters- und entwicklungsangepassten Regeln für Kinder und Jugendliche müssen die Menschenrechtscharta, insbesondere die Kinderrechtscharta [22], und die Convention on the Rights of Persons with Disabilities [23] sein. Minimale Versorgungsstandards in diesem Bereich umfassen die verpflichtende Schulung in Deeskalation sowie die Schaffung eines sicheren, therapeutischen Milieus (architektonisch und atmosphärisch) mit Traumapädagogischem Know-how und einer Beziehungsorientierten Versorgung. Weiterhin muss eine externe Kontrolle durch die Bezirksgerichte respektive durch Institutionen wie die OPCAT (siehe [6] in diesem Heft) erfolgen.

Gerade unter historischen Gesichtspunkten ist der Kooperation mit der Kinder- und Jugendhilfe besonderes Augenmerk zu schenken. In der Nazizeit und in der Nachkriegszeit waren beide Systeme in die menschenunwürdige Behandlung von Kindern und Jugendlichen involviert. Umso mehr ist es daher wichtig sich täglich zu fragen, inwieweit die Kinderund Jugendpsychiatrie durch gesellschaftliche Bedingungen gezwungen wird, bestimmte Maßnahmen zu übernehmen, obwohl sie entweder gar nicht zuständig ist (z. B. bei pädagogischen oder sozialen Krisen) oder sie allgemeine Mangelzustände wie das Fehlen qualitätsvoller Einrichtungen für psychisch kranke Kinder und Jugendliche kompensieren muss. Es gibt in Österreich funktionierende Beispiele guter Kooperation zwischen der Kinder- und Jugendpsychiatrie und der Kinder- und Jugendhilfe, die jeweils auch noch verbessert, ausgebaut und vor allem flächendeckend etabliert werden können (Kindernetzwerk Industrieviertel Niederösterreich [20] oder Krisenmanual Salzburg [19]).

Eine ausführliche und umfassende Darstellung der Geschichte der österreichischen Kinder- und Jugendpsychiatrie ist noch ausständig wie auch die Auseinandersetzung mit den Folgen für die betroffenen Menschen bisher erst teilweise erfolgt ist [8]. Diesen Menschen gilt bei der Bearbeitung dieses Themas insbesondere unsere Anteilnahme und eine Entschuldigung pars pro toto.

An dieser Stelle sei auch einem österreichischen Kinder- und Jugendpsychiater besonders gedankt: Prof. Dr. Ernst Berger, ehemals Vorstand der Abteilung für Kinder- und Jugendpsychiatrie und Behindertenmedizin am Rosenhügel. Er hat als Nachfolger A. Rett's die Auswirkungen der Rett'schen Arbeit direkt erlebt und die Abteilung zu einer modernen kinderund jugendpsychiatrischen Abteilung umgeformt. Der Dank gilt ihm aber vor allem für sein Engagement in der Aufarbeitung der Geschichte der Kinder- und Jugendpsychiatrie in Österreich, das sich in vielen Publikationen zu diesem Thema und in seiner Arbeit für die Menschenrechtskommission niederschlägt.
Acknowledgements Open access funding provided by Paracelsus Medical University.

Interessenkonflikt L. Thun-Hohenstein gibt an, dass kein Interessenkonflikt besteht.

Open Access Dieser Artikel wird unter der Creative Commons Namensnennung 4.0 International Lizenz (http:// creativecommons.org/licenses/by/4.0/deed.de) veröffentlicht, welche die Nutzung, Vervielfältigung, Bearbeitung, Verbreitung und Wiedergabe in jeglichem Medium und Format erlaubt, sofern Sie den/die ursprünglichen Autor(en) und die Quelle ordnungsgemäß nennen, einen Link zur Creative Commons Lizenz beifügen und angeben, ob Änderungen vorgenommen wurden.

\section{Literatur}

1. Asperger H. Heilpädagogik. Wien New York: Springer; 1968. 2. Asperger H. Die „Autistischen Psychopathien“im Kindesalter. Arch Psychiatr Nervenkr (1970). 1994;117:76-136.

3. Asperger-Felder M. Zum Sehen geboren, zum Schauen bestellt.In:PollakA (Hrsg.).Aufden Spuren Hans Aspergers. Stuttgart: Schattauer; 2015. S.38-43.

4. Aus der Schmitten I. Schwachsinnig in Salzburg. Zur Geschichte einer Aussonderung. Salzburg: Umbruch; 1985.

5. Berger E. Die österreichische Kinder- und Jugendpsychiatrie nach 1945 bis 1975. In: Fangerau H, Topp S, Schepker K(Hrsg.). Kinder- und Jugendpsychiatrie im Nationalsozialismus und in der Nachkriegszeit. Berlin: Springer; 2017.

6. Berger E. Menschenrechtsmonitoring im Kinder- und Jugendbereich. Neuropsychiatrie. 2017;31:3. doi:10.1007/ s40211-017-0244-z.

7. Berger E, Aichhorn W, Friedrich MH, Fiala-Preinsberger S, Leixnering W, Mangold B, Spiel G, Tatzer E, Thun-Hohenstein L. Kinder- und Jugendpsychiatrische Versorgung in Österreich. Neuropsychiatrie.2006;2:86-90.

8. Brainin E, Teicher S. Terror von außen am Beispiel Spiegelgrund: Traumatische erfahrungen in der Kindehit und deren Folgen. Prax Kinderpsychol Kinderpsychiatr. 2009;58(7):530-52.

9. Brezinka W. Heilpädagogik an der Medizinischen Fakultät der Universität Wien: ihre Geschichte von 1911-1985. ZPädagog. 1997;43(3):395-420.

10. Bundesministerium für Gesundheit und Frauen. Ärzteausbildungsordnung 2006. Wien: Bundesministerium für Gesundheit und Frauen; 2006.

11. BundesministeriumfürGesundheit. Ärzteausbildungsordnung 2015 - Fassung vom 17.4.2017. Berlin: Bundesministerium für Gesundheit; 2017.

12. Castell R. Begründer der Kinder- und Jugendpsychiatrie. In: Castell R, Nedoschill J, Rupps M, Bussiek D (Hrsg.). Geschichte der Kinder- und Jugendpsychiatrie in Deutschland in den Jahren 1937 bis 1961. Göttingen: Vandenhoeck \&Ruprecht;2003.S.397-496.

13. Castell R. Die wissenschaftlichen Kongresse bis 1945 und die Gründung und Entwicklung der Deutschen Fachgesellschaft. In: Castell R, Nedoschill J, Rupps M, Bussiek D (Hrsg.). Geschichte der Kinder- und Jugendpsychiatrie in Deutschland in den Jahren 1937 bis 1961. Göttingen: Vandenhoeck\& Ruprecht;2003.S.4-87.

14. Castell R. Geschichte der Kinder- und Jugendpsychiatrie in Deutschland in den Jahren 1937-1961. Göttingen: Vandenhoeck\& Ruprecht; 2003.

15. Castell R. Vorgeschichte und Entstehung der Kinderabteilung der Nervenklinik in Tübingen 1919/20 im Spannungsfeld zwischen therapeutischer Innovation und sozia- 
ler Selektion. In: Castell R, Nedoschill J, Rupps M, Bussiek D (Hrsg.). Geschichte der Kinder- und Jugendpsychiatrie in Deutschland in den Jahren 1937 bis 1961. Göttingen: Vandenhoeck\& Ruprecht;2003. S. 13-8.

16. Castell R. Einleitung. In: Castell R (Hrsg.). Hundert Jahre Kinder- und Jugendpsychiatrie. Göttingen: V\&R unipress; 2008. S. 9-15.

17. Czech H. Hans Asperger und die „Kindereutanasie“ in Wien - mögliche Verbindungen. In: Pollak A (Hrsg.). Auf den Spuren Aspergers. Stuttgart: Schattauer; 2013. S. 24-9.

18. Czech H. Der Spiegelgrund-Komplex Kinderheilkunde, Heilpädagogik, Psychiatrie und Jugendfürsorge im Nationalsozialismus. Österr Z Geschichtswiss. 2014;25(1+2): 194-219.

19. Elmer R., Thun-Hohenstein L. Krise als Chance - Handlungsleitfaden für die KrisenarbeitmitKindernund Jugendlichen im Bundesland Salzburg. Land Salzburg, Abteilung 3 02.2015 .

20. Fliedl R. Krisenmanual für Kinder und Jugendliche im Industrieviertel. Hinterbrühl: Karl Landsteiner Gesellschaft; 2017.

21. Gabriel E. NS-Euthanasie in Österreich von 1938-1945. psychopraxis.neuropraxis. 2016;19:21-24.

22. General Assembly of United Nations. UN-Kinderrechtskonvention-Übereinkommen über die Rechte des Kindes. 1989.

23. General Assembly of United Nations. Convention on the rights of persons with disabilities (CRPD). 2006.

24. Haack K, Kumbier E. Verbrechen an Kindern und Jugendlichen in der NS-Zeit. Z Kinder Jugendpsychiatr. 2013;41(Sonderheft):12-9.

25. Hamburger F. Die Neurosen des Kindesalters. Stuttgart: FerdinandEnke; 1939.

26. Hartl C. Der Kinder- und Jugendpsychiater in der Praxis: ein Primärversorger? Pädiatr Pädol.2013;48(Suppl1):91-8.

27. Hartl C, Karwautz A. Zehn Jahre Kinder- und Jugendpsychiatrie in Österreich: ein neues ärztliches Sonderfach in den Strukturen des Gesundheitswesens. Neuropsychiatrie. 2017;31(3). doi:10.1007/s40211-017-0235-0.

28. Häßler F, Reis O. Die Erinnerung an die Geschichte als Mahnung für die Gegenwart. Z Kinder Jugendpsychiatr Psychother.2013;41(Sonderheft):25-7.

29. Kanner L. Child psychiatry. Springfieeld IL: C.C. Thomas; 1948.

30. Key E. Das Jahrhundert des Kindes. Berlin: Fischer; 1902.

31. Kuszen P. Sonderformen der Intelligenz. In: Stur O (Hrsg.). 2nd International Congress on Mental Retardation, Vienna 1961: Proceedings, Part II. Basel: Karger; 1963. S. 17-21.

32. Madlener E. Wunderblock. Eine Geschichte der modernen Seele. Katalog zur Ausstellung. Wien: Seitenberg; 1989.

33. Malina P. Zur Geschichte des „Spiegelgrundes“. In: Berger E (Hrsg.).Verfolgte Kindheit. Wien:Böhlau;2007.S. 159-92.

34. Mayrhofer H, Wolfgruber G, Geiger K, Hammerschick W, ReidingerV.Kinderund JugendlichemitBehinderunginder Wiener Psychiatrie 1945-1989: stationäre Unterbringung am Steinhof und Rosenhügel. Schr Rechts Kriminalsoziol. 2017;8:335-528.
35. Neugebauer W. Die Klinik „AmSpiegelgrund“ 1940-1945, eine „Kinderfachabteilung“ im Rahmen der NS „Euthanasie“.Wien: Gemeinde Wien; 1997.

36. Neumärker K-J. Leo Kanner. In: Castell R (Hrsg.). Hundert Jahre Kinder- und Jugendpsychiatrie. Göttingen: V\&R unipress; 2008. S. 47-72.

37. Poustka F. Autismus-Spektrum Störungen - Hans Asperger und klinische Aspekte heute. In: Pollak A (Hrsg.). Auf den Spuren Aspergers. Stuttgart: Schattauer;2009. S. 1-14.

38. Ralser M, Sieder R. Die Kinder des Staates. Österr Z Geschichtswiss. 2014;25(1):7-17.

39. Ronen GM, Meaney B, Dan B, Zimprich F, Stögmann W, Neugebauer W. From eugenic euthanasia to habilitation of "disabled" children: Andreas Rett's contribution. J Child Neurol.2009;24(1):115-27.

40. Rudolph C, Benetka G. Zur Geschichte des Wiener Jugendamtes. In: Berger E (Hrsg.). Verfolgte Kindheit. Wien: Böhlau;2007.S. 47-88.

41. Schepker K, Fangerau H. Die Gründung der Deutschen Gesellschaft für Kinderpsychiatrie und Heilpädagogik: Der Weg von Paul Schröder zum Gründungsvorsitzenden. Z Kinder Jugendpsychiatr Psychother. 2016;44(3):180-8.

42. Schorske CE. Österreichische Kultur 1870-1914, Betrachtungen eines Historikers. In: Waissenberger R (Hrsg.). TraumundWirklichkeitWien 1870-1930.Wien:Eigenverlag der Museen der Stadt Wien; 1985. S. 12-25.

43. Schreiber A. Das Buch vom Kinde. Leipzig Berlin: Teubner; 1907.

44. Sousek R. Hans Asperger (1906-1980) - Versuch einer Annäherung. In: PollakA (Hrsg.). Aufden Spuren Hans Aspergers. Stuttgart: Schattauer;2013.S. 15-23.

45. Sperk G, Dietrich-Daum E, Ralser M, Schreiber H, Gerstgrasser P, Purtscher-Penz AK, Berger E. Die Innsbrucker Beobachtungsstation von Maria Nowak-Vogl. Innsbruck: Arnold Digitaldruck; 2015.

46. Spitzy H. Die körperliche Erziehung des Kindes. Berlin Wien: Urban \& Schwarzenberg; 1914.

47. Tatzer E, Krisch K, Hanich R. Arbeit mit psychisch gestörten Eltern in einer kinderpsychiatrischen Einrichtung. Prax Kinderpsychol Kinderpsychiatr. 1994;43(5):170-4.

48. Tatzer E, Schubert MT. Systemtherapie im Kinderheim. In: Brunner EJ, Reiter L, Reiter-Theil S (Hrsg.). Von der Familientherapie zur Systemischen Perspektive. Berlin Heidelberg: Springer; 1988. S. 143-54.

49. Teller S. Zur Heilpädagogisierung der Strafe: Zur Geschichte der Wiener Jugendgerichtshilfe von 1911-1928. 2009. University of Vienna, Abteilung für Pädagogik.

50. Toth B. Die sind wirklich in der Hölle gelandet. Falter. 2017;11:10-3

51. Waibel A. Prof. Dr. Otto Löwenstein und die Gründerjahre der Provinzkinderanstalt für seelisch abnorme: 1926-1933. Köln Bonn: Rheinland Verlag; 1998. Dissertation.

52. Waissenberger R. Traum und Wirklichkeit Wien 1870-1930. Wien: Eigenverlag der Museen der Stadt Wien; 1985.

53. Warnke A, Lehmkuhl G. Kinder- und Jugendpsychiatrie und Psychotherapie in Deutschland. Stuttgart: Schattauer; 2011. 